\title{
Study on fish diversity and need for their conservation of Bakara River, Morang district, eastern Nepal
}

\author{
Shiva Narayan Yadav \\ Department of Zoology, M.M.A.M. Campus, T.U., Bhadrapur, Nepal \\ *E-mail:yadav_s67@yahoo.com
}

\begin{abstract}
Forty-eight fish species were collected in the present study. The population status of important fishes Chagunius chagunio and Channa striatus were found to be threatened. Mastacembulus puncalus was vulnerable and Clupisoma garua and Puntius ticto threatened because of pollution.
\end{abstract}

Key words: Threatened fishes, population status, Chagunius chagunio.

\section{Introduction}

Bakara river is a large river of Morang district. It originates from Mikjani hill of "Mahabharat Lekh" and flows towards south through the middle of Morang district. It crosses Takuwa and Rangeli Municipality making a wavy bend and enters Bihar state of India.

Bakara river had rich fish diversity and other aquatic bioresources before 20 years, which are now rapidly deteriorating. Major causes are the human population explosion, contamination with agro-chemicals, deforestation, and over-fishing and indiscriminate fishing (Bhagat, 1985; John \& Dhewajoo, 1989; Pokharel, 1998, 1999; Jayaram, 1999)

\section{Materials and Methods}

Fishes were collected at monthly intervals from five sites of Bakara river during August, 2014 and August, 2015. Fish collections were made with the assistance of local fishermen using gill nets and other devices. The collected fishes were preserved in 5-8\% formaldehyde solution and brought to the zoology Laboratory. The threatened species were released immediately into water after collection.

Identification of fishes were done with the help of standard literature (Shrestha, 1981, 1990, 1994). Information on fish diversity, their habit, habitat and population status were collected from local fishermen, local people and through field observation. Brief notes on the ecological features, size, colouration and local names were noted down in the field itself. The criteria laid out by International Union for Conservation of Nature and Natural resources, IUCN (1994) was followed for assessment of the status of fishes.

\section{Results and Discussion}

Fishes collected from Bakara river belonged to 7 orders, 18 families, 28 genera, and 48 species (Table 1). The abundant species were Puntius sophore, P. chola, P. conchonius, Mystusbleekeri, Xenentodon cancila, Labeo rohita, Mastacembelus armatus, Esomus danricus, Channa marulius, Wallago attu, Ompok bimaculatus. Similarly, the species 
which were moderately distributed in 4 and 5 sites and having less abundance were Chagunius chagunio, Gudusia chapra, Channa stewartii, Labeo angra. But the species distributed only in 1 and 2 sites and having much less abundance were Channa punctatus, Clarias batrachus, Puntius sarana, Clupiso magarua. The freshwater Sisor rhabdophorus and Gagata cenia were recorded before 25 years, but could not be found during the present study period. In general, the habitat condition for fishes in Bakra river appears favouable at present, however the river is found to be disturbed due to the lack of proper knowledge and management. It has been influenced by land-slides, soil erosion floods and siltation mostly due to deforestation. The hotels, restraurants, shop and residence buildings are situated near the bank of Bakara river which is polluting due to discharge of wastes and domestic sewage directly into it without any pre-treatment. The agriculture activities near bank of river are also affecting the fish resources.

Deforestation was recorded at many places between Takuwa and Rangeli municipality near the bank of Bakara river causing soil erosion, floods and increase in turbidity which affect the natural properties of water and also affect the components of the aquatic ecosystem. Release of toxic substances in the form of chemical fertilizers and pesticide through surface run-off from the agricultural fields near the bank of river and use of soap and detergents by human activities affect upon the physiochemical and biological properties of water and ultimately affect the biotic components of the aquatic ecosystem. Similarly, the construction of dams on Bakara river is found to prevent the free movement of fishes which move upstream for breeding purposes and indiscriminate fishing (fry to adult) including brood fishes during their breeding season and fishes having depleted population are also threatened.

Table 1. Fish diversity and distribution in Bakara River.

\begin{tabular}{|c|c|c|c|c|c|c|}
\hline \multirow{2}{*}{ S.N. Systemic position/Scientific name } & \multirow{2}{*}{ Local name } & \multicolumn{5}{|c|}{ Distribution Sites Remarks } \\
\hline & & & B & $\mathrm{C}$ & D & $\mathbf{E}$ \\
\hline \multicolumn{7}{|c|}{ Order : Cypriniformes, Family : Cyprinidae, Subfamily : Cyprinae } \\
\hline \multicolumn{7}{|l|}{ Genus : Catla } \\
\hline \multicolumn{7}{|l|}{ Genus: Chagunius } \\
\hline 2. Chagunius chagunio & Chaguni & $x$ & $\sqrt{ }$ & $\sqrt{ }$ & $x$ & $\times$ \\
\hline \multicolumn{7}{|l|}{ Genus : Cisshinus } \\
\hline 3. Cisshinus mrigala & Mrigal(Naini) & $\sqrt{ }$ & $\sqrt{ }$ & $\sqrt{ }$ & $\sqrt{ }$ & $\sqrt{ }$ \\
\hline 4. Cisshinus reba & Rewa & $\sqrt{ }$ & $\sqrt{ }$ & $\sqrt{ }$ & $\sqrt{ }$ & $\sqrt{ }$ \\
\hline \multicolumn{7}{|l|}{ Genus : Labeo } \\
\hline 5. Labeo rohita & Rohu & $\sqrt{ }$ & $\sqrt{ }$ & $\sqrt{ }$ & $\sqrt{ }$ & $\sqrt{ }$ \\
\hline 6. L. angra & Boga & $\sqrt{ }$ & $\sqrt{ }$ & $x$ & $\sqrt{ }$ & $\mathrm{x}$ \\
\hline 7. L. calbasu & Kalbasu & $x$ & $x$ & $\sqrt{ }$ & $\sqrt{ }$ & $\sqrt{ }$ \\
\hline 8. L. gonius & Kursa & $\sqrt{ }$ & $\sqrt{ }$ & $x$ & $\sqrt{ }$ & $x$ \\
\hline 9. L. dero & Gundi & $\times$ & $\sqrt{ }$ & $\sqrt{ }$ & $\times$ & $\sqrt{ }$ \\
\hline \multicolumn{7}{|l|}{ Genus : Puntius } \\
\hline 10. Puntius sophore & Pothi & $\sqrt{ }$ & $\sqrt{ }$ & $\sqrt{ }$ & $x$ & $\sqrt{ }$ \\
\hline 11. P. ticto & Sidre & $\sqrt{ }$ & $\sqrt{ }$ & $x$ & $x$ & $x$ \\
\hline 12. P. chola & Pothiya & $\sqrt{ }$ & $\sqrt{ }$ & $\sqrt{ }$ & $\sqrt{ }$ & $x$ \\
\hline 13. P. conchonius & Pothi, sidre & $\sqrt{ }$ & $\sqrt{ }$ & $\sqrt{ }$ & $x$ & $\sqrt{ }$ \\
\hline
\end{tabular}


14. P. sarana

Bhitti

$\begin{array}{lllll}\sqrt{ } & \sqrt{ } & \sqrt{ } & \sqrt{ } & \sqrt{ }\end{array}$

Genus : Esomus

15. Esomus danricus

Darai $\sqrt{ } \times \times \times$

Family : Cobitidae, Subfamily : Nemacheilinae

Genus : Acanetrocotitis

16. Acanthocobitis botia(Nemacheilus botia) Baghe

Genus: Botia

17. Botia lohachata

Getu

$\sqrt{ } \times \sqrt{ } \times \sqrt{ }$

Order : Siluriformes, Family: Bagridae

Genus: Mystus

18. Mystus bleekeri

19. M. tegra

20. M. mehoda

21. M. aor

22. M. cavasius

23. M. vittatus

24. M. seenghala

$\begin{array}{lccccc}\text { Tengra } & \times & \sqrt{ } & \sqrt{ } & \times & \sqrt{ } \\ \text { Tengri } & \times & \times & \sqrt{ } & \sqrt{ } & \sqrt{ } \\ \text { Belauni } & \sqrt{ } & \times & \sqrt{ } & \times & \times \\ \text { Kanti } & \times & \times & \sqrt{ } & \sqrt{ } & \sqrt{ } \\ \text { Junge } & \sqrt{ } & \sqrt{ } & \times & \times & \sqrt{ } \\ \text { Tengra } & \sqrt{ } & \sqrt{ } & \sqrt{ } & \sqrt{ } & \sqrt{ } \\ \text { Kanti } & \sqrt{ } & \sqrt{ } & \sqrt{ } & \times & \times\end{array}$

Family : Siluridae

Genus: Ompok

25. Ompok bimaculatus

Genus : Wallago

26. Wallago attu

Papta

$\sqrt{ } \sqrt{ } \quad \sqrt{ } \quad \sqrt{ } \times$

Family: Schilbeidae

Genus: Clupisoma

27. Clupisoma garua

Jalkapoor $\sqrt{ } \times \sqrt{ } \times \quad \times$

Genus: Eutropiichthys

28. Eutropiichthys vacha

Bohari

$\begin{array}{lllll}\sqrt{ } & \sqrt{ } & \sqrt{ } & \sqrt{ } & \sqrt{ }\end{array}$

Genus: Pseudetropius

29. Pseudetropius atherinoides

Bachawa $\sqrt{ } \sqrt{ } \sqrt{ } \times \times$

Family: Clariidae

Genus:Clarias

30. Clarias batrachus

Mungri

$\sqrt{ } \sqrt{ } \quad \sqrt{ } \quad \sqrt{ } \sqrt{ }$

Family: Heteropneustidae

Genus: Heteropneustes

31. Heteropneustes fossilis

Singhi

$\begin{array}{lllll}\sqrt{ } & \sqrt{ } & \sqrt{ } & \sqrt{ } & \sqrt{ }\end{array}$

Order: Beloniformes, Family: Belonidae,

Genus: Xenentodon

32. Xenentodon cancila

Kauwo

$\begin{array}{lllll}\sqrt{ } & \sqrt{ } & \sqrt{ } & \sqrt{ } & \sqrt{ }\end{array}$

Order: Symbranchiformes, Family: Symbranchidae

Genus: Monopterus (Amphipnous)

33. Monopterus cuchia

Bam

$\sqrt{ } \sqrt{ } \times \times$

Family: Mastacembelidae

Genus: Macrognathus

34. Macrognathus pancalus

35. M. aculeatus

$\begin{array}{lllllllllll}\text { Kath gainchi } & \sqrt{ } & \sqrt{ } & \sqrt{ } & \times & \sqrt{ }\end{array}$

Genus: Mastacembelus

36. Mastacembelus sarmatus

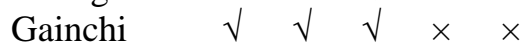

$\begin{array}{llllll}\text { Chusi Bam } & \sqrt{ } & \sqrt{ } & \sqrt{ } & \sqrt{ } & \sqrt{ }\end{array}$ 
Order: Perciformes, Family: Ambassidae,

Genus: Chanda

37. Chanda nama

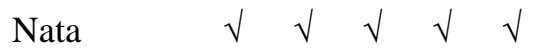

Family: Nandidae,

Genus: Nandus

38. Nandus nandus

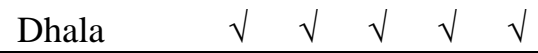

Family: Gobiidae,

Genus: Glossogobius

39. Glossogobius giuris

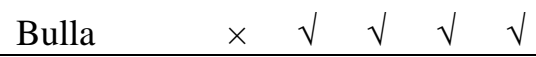

Family: Anabantidae,

Genus: Anabas

40. Anabas testudineus

\begin{tabular}{llllll} 
Kabai & $\sqrt{ }$ & $\sqrt{ }$ & $\sqrt{ }$ & $\sqrt{ }$ & $\sqrt{ }$ \\
\hline
\end{tabular}

Family: Belontiidae,

Genus: Colisa

41. Colisa fasciatus

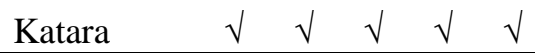

Family: Channidae,

Genus: Channa

42. Channa marulius

43. Channa orientalis (gachua)

44. C. panctatus

45. C. stewartii

46. C. striatus

Order: Clupiformes, Family: Clupeidae

Bhaura $\sqrt{ } \quad \sqrt{ } \quad \sqrt{ } \times \times$

$\begin{array}{llllll}\text { Chenga } & \sqrt{ } & \sqrt{ } & \sqrt{ } & \sqrt{ } & \sqrt{ }\end{array}$

$\begin{array}{llllll}\text { Garahi } & \sqrt{ } & \sqrt{ } & \sqrt{ } & \sqrt{ } & \sqrt{ }\end{array}$

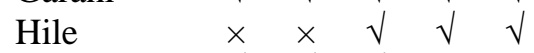

Saura

$\begin{array}{lllll} & \sqrt{ } & \sqrt{ } & \times & \times\end{array}$

Genus: Gudusia

47. Gudusia chapra

Suiya $\quad \sqrt{ } \times \quad \times \quad \times$

Order: Osteoglossiformes, Family: Notopteridae

Genus: Notopterus

48. Notopterus notopterus

Golhai

$\begin{array}{llllll} & \sqrt{ } & \sqrt{ } & \sqrt{ } & \sqrt{ }\end{array}$

\section{Conclusions}

Bakara river has a rich fish diversity, however, the Bakra river is being disturbed due to cutting down of forests, unusual erosion, indiscriminate fishing and the harmful use of toxic substances. It requires strict rules and regulations to check such disturbances.

\section{Acknowledgements}

Author is very much thankful to local people of Takuwa and Rangeli Munacipality, Morang district for their sincere help during the survey of fishes.

\section{Reference}

Bhagat, R.P. 1985. Fish and fishery resources of Morang district, Koshi zone, Nepal. Central Department of Zoology., T.U., Kirtipur. (M.Sc. Desertation)

IUCN, 1994. IUCN Red list categories. Gland Switzerland.

Jayaram, K.C. 1999. The freshwater fishes of Indian region. Narendra Publishing Co., New Delhi, India. 
John, A. \& R.G. Dhewajoo 1989. Effect of ecological parameters on fishes of Pokhara valley. A report submitted to Nepal Academy of Science and Technology (NAST), Kathmandu, Nepal 88p.

Pokharel, K.K. 1998. Fish diversity and feeding habits of fishes of lakes situated in Pokhara Valley. A report summited to UGC.

Pokharel, K.K. 1999. Phewa lake as fishery resources and its conservation. Pragya Manch. Academic Forum 12(7): 76-80.

Shrestha, J. 1981. Fishes of Nepal. Curriculum Development Centre, T.U., Kathmandu, Nepal.

Shrestha, J. 1994. Fish species in different water bodies of Nepal and their need for conservation. Ecoprint (1): 7-19.

Shrestha, T.K. 1990. Resource ecology of the Himalayan waters. Curriculum Development Centre, T.U., Kathmandu, Nepal. pp. 170-171. 